\title{
Ubiquitin E3 Ligase A20 Contributes to Maintaining Epithelial Barrier Function
}

\author{
Peixin Huang ${ }^{\mathrm{a}}$ Xiao-Rui Geng ${ }^{\mathrm{b}} \quad$ Gui Yang $^{\mathrm{b}} \quad$ Chi Chen $^{\mathrm{a}}$ Zhanju Liu ${ }^{\mathrm{a}}$ \\ Ping-Chang Yang ${ }^{b}$ \\ ${ }^{a}$ Department of Gastroenterology, the Shanghai Tenth People's Hospital, Tongji University, Shanghai \\ ${ }^{b}$ Department of Pathology \& Molecular Medicine, McMaster University, Hamilton, ON, Canada
}

\section{Key Words}

Epithelial barrier $\cdot$ Allergen • Ubiquitin E3 ligase • Endosome • Lysosome

\begin{abstract}
Background and Aims: Epithelial barrier dysfunction is involved in the pathogenesis of allergic diseases; the mechanism is to be further understood. Ubiquitin E3 ligase A20 (A20) plays a role in maintaining the homeostasis in the body. This study aims to investigate the role of A20 in maintaining the epithelial barrier function. Methods: Human intestinal epithelial cell line, Caco-2 cells, was cultured to monolayers to test the endocytosis and degradation of a model allergen, ovalbumin (OVA). The role of A20 in the endosome/lysosome fusion in epithelial cells was tested with A20-sufficient and A20-deficient Caco-2 cells and visualized by immunocytochemistry. Results: Caco-2 cells could endocytose exogenous allergens (OVA) in culture. The endocytic OVA was degraded in A20-sufficient Caco-2 cells via the mechanism of endosome/lysosome fusion, while the A20-deficient Caco-2 monolayers converted the OVA to the basal compartment of transwells, which conserved the antigenicity reflected by that it induced $T$ cell proliferation in an allergen-specific manner. A20 was required in the fusion of endosomes and lysosomes. Conclusion: A20 contributes to maintaining the epithelial barrier function.
\end{abstract}

Copyright $\odot 2012$ S. Karger AG, Basel

\section{Introduction}

The gut epithelial barrier consists of epithelial cells and the tight junctions. The barrier physically separates the gut tissue from the harsh luminal environment [1]. The epithelial cells also absorb water and nutrients from the luminal digested food [2]. Although there 
are protein enzymes in the lumen to digest the ingested proteins, some protein molecules and peptides with antigenicity are absorbed by epithelial cells; specifically, in the intestine suffering from allergic disorders, a large amount of allergens can be absorbed by the epithelial cells and delivered to the subepithelial region to contact immune cells, to initiate skewed immune responses and trigger immune inflammation attacks $[3,4]$. The underlying mechanism is not fully elucidated yet.

After endocytosis, the allergens can be wrapped by the plasma membrane to form endosomes in epithelial cells. The allergen-carrying endosomes then fuse with lysosomes where the allergens may be digested by the acid hydrolase enzymes [3]. It implies that the fusion of endosome and lysosome is a critical step to digest the endocytic allergens. The endosome and lysosome fusion has been studied extensively $[5,6]$. The disturbance of the fusion of endosome and lysosome can interfere with the degradation of the endocytic allergens [7]. Yet, whether the interference of the endosome/lysosome fusion is associated with allergic disorders has not been fully understood.

Ubiquitination is essential for transporting the endocytic cargo to lysosomes. Published data indicate that ubiquitination is one of the major determinants in the vacuolar trafficking in the cell [8], which is required for sorting endocytic cargo to lysosomes [9]. Ubiquitin E3 ligase A20 (A20, in short) plays an important role in the regulation of immune responses and epithelial barrier function [10,11]. A20 is also involved in the fusion of endosome to lysosome [12]. In this study, we found that A20 plays an important role in the endosome/ lysosome fusion that determined the degradation of the endocytic allergens in gut epithelial cell line, Caco-2 cells.

\section{Materials and Methods}

Reagents

The ovalbumin (OVA) was purchased from Sigma Aldrich (Shanghai, China). Antibodies of OVA, EEA1, LAMP2, A20, and A20 shRNA were purchased from Santa Cruz Biotech (Shanghai, China). Immune cell isolation kits were purchased from Miltenyi Biotech (Shanghai, China).

Cell culture

Caco-2 cell line (passage 35-45; ATCC, Manassas, VA) was cultured in Dulbecco's Modified Eagle Medium, supplemented with $10 \%$ fetal bovine serum, $100 \mathrm{units} / \mathrm{ml}$ penicillin and $100 \mathrm{units} / \mathrm{ml}$ streptomycin and $1 \mathrm{mM}$ non-essential amino acids in a humidified $\mathrm{CO}_{2}(5 \%)$ incubator at $37^{\circ} \mathrm{C}$. Culture medium was replaced every other day for 2 weeks, and then daily thereafter for the next 1 week until the formation of the monolayer.

\section{Assessment of trans-epithelial permeability}

Caco- 2 cells were seeded in 12-well Transwell ${ }^{\circledR}$ inserts (Millipore Corp., Bedford, MA; pore size: $0.4 \mu \mathrm{m}$; surface area: $1.12 \mathrm{~cm}^{2}$ ) at $1 \times 10^{6}$ cells $/ \mathrm{cm}^{2}$. After reaching the confluence (TER $\geq 500 \Omega \mathrm{cm}^{2}$ ), the OVA was added to the apical chambers at the indicated concentrations. Samples were taken from the basal chambers at indicated time points and analysed by ELISA. TER was recorded before and $24 \mathrm{~h}$ after the addition of allergens.

\section{Cellular protein extraction}

Caco- 2 cells were harvested from the inserts by $0.05 \%$ trypsin-EDTA. Total proteins were extracted from the cells. Briefly, $0.2 \mathrm{ml}$ cell lysis buffer [20mM Tris- $\mathrm{HCl}$ (pH 7.5), 150mM NaCl, 1mM EDTA, 1mM EGTA, $1 \%$ Triton $\mathrm{X}-100,2.5 \mathrm{mM}$ sodium pyrophosphate, $1 \mathrm{mM}$ beta-glycerophosphate, $1 \mathrm{mM} \mathrm{Na}_{3} \mathrm{VO}_{4}, 10 \mathrm{mM} \mathrm{NaF}$, $1 \mathrm{mM}$ PMSF, $1 \mu \mathrm{g} / \mathrm{ml}$ leupeptin, and $0.3 \mu \mathrm{M}$ aprotinin, $130 \mu \mathrm{M}$ bestatin, $14 \mu \mathrm{M}$ E-64] was added to $10^{7}$ cells in an Eppendorf's tube. Placing the tube on ice, the cells were passed through an 18 gauge needle for 10 times; the tube remained on ice for $30 \mathrm{~min}$. The tube was centrifuged $(\times 14000 \mathrm{~g})$ for $20 \mathrm{~min}$ at $4^{\circ} \mathrm{C}$. The supernatants were collected; the concentration of protein was determined by Bio-Rad protein assay. 


\section{Concentrating protein from culture supernatants}

The transported OVA through Caco-2 cell monolayer in the culture supernatant at the basal chamber was concentrated by the saturated ammonium sulphate precipitation. The culture supernatants were collected from the basal chambers to concentrate the proteins by saturating ammonium sulphate precipitation following the standard operating procedures in our laboratory that was also published elsewhere [13].

\section{Enzyme-linked immunoassay (ELISA)}

The contents of A20 and OVA from the collected samples were determined by ELISA. A 96-well plate was coated with diluted standard proteins or sample protein $(20 \mu \mathrm{g} / \mathrm{well} ; 0.1 \mathrm{ml} /$ well was used throughout the experiment) and incubated at $4^{\circ} \mathrm{C}$ overnight. The plated was blocked by adding $5 \%$ skim milk for $30 \mathrm{~min}$; the first antibodies $(10 \mathrm{ng} / \mathrm{ml})$ were added, incubated for $1 \mathrm{~h}$ and followed by the HRP-conjugated second antibodies $(5 \mathrm{ng} / \mathrm{ml})$, incubated for $1 \mathrm{~h}$. The immune complexes were detected by addition of Tetramethylbenzidine (TMB) for $15 \mathrm{~min}$. Three wash steps with TBST (Tris $10 \mathrm{mM}, 0.09 \% \mathrm{NaCl}, 0.2 \%$ Tween 20) were performed after incubation with first and second antibodies. The sample's optical density was read with a microplate reader (BioTek, FL800; Shanghai, China). The concentrations of the targeted proteins were calculated against the standard curve. All samples were added in duplicate. The negative control wells were added with isotype IgG to replace the first antibody. The OD values from the negative control wells were subtracted from all readings.

\section{Immunocytochemistry}

Caco-2 cells were collected from transwell inserts with trypsin-EDTA; the single cell suspension was made and fixed with $2 \%$ paraformaldehyde for $2 \mathrm{~h}$. Following our published procedures [14] with modifications, the cells were stained with antibodies $(1 \mu \mathrm{g} / \mathrm{ml}$ ) of the OVA, EEA1 (an endosome marker) and LAMP2 (a lysosome marker) in an Eppendorf tube for $1 \mathrm{~h}$; then, fluorescence labelled second antibodies $(1 \mu \mathrm{g} / \mathrm{ml})$ was added and incubated for $1 \mathrm{~h}$. Washing with PBS for 3 times was performed after incubation with antibodies. The cells were smeared onto a slide, mounted with a cover slip with anti-fade media. The slides were observed under a confocal microscope with the $\times 630$ objective. The positive staining part was further enlarged with the built-in "enlarge feature" and photographed when it was appropriate.

\section{Image analysis}

In each cell, the positive staining particles of OVA, A20, EEA1 and LAMP2 were counted on the photographs. The merged colors were sorted respectively and counted. Thirty cells were analysed for each group. The slides and photographs were coded. The observer was not aware of the code to avoid the observer bias.

\section{RNA interference}

Some Caco-2 cells were transduced with shRNA of A20 (or control shRNA using as controls) to knock down the A20 gene following the manufacturer's instruction.

\section{Western blotting}

The effect of RNA interference was revealed by Western blotting. The cellular extracts were fractioned in SDS PAGE and transferred onto a nitrocellulose membrane. The membrane was blocked with 5\% skim milk, incubated with anti-A20 antibody $(100 \mathrm{ng} / \mathrm{ml})$ and followed by the HRP-labelled second antibody $(50 \mathrm{ng} / \mathrm{ml})$. The immune complexes were revealed with the enhanced ECL kit.

\section{Assessment of antigen-specific $T$ cell activation}

Ten patients ( 5 male, 5 female, age: 24-56 years old) with egg allergy history, serum egg-specific IgE was greater than $30 \mathrm{ng} / \mathrm{ml}$, were recruited to this study. The using human tissue in the study was approved by the Human Study Ethic Committee at Tongji University. Forty milliliter blood was obtained from each patient. The peripheral blood mononuclear cells were isolated by the gradient density concentration; $\mathrm{CD}^{+}$ $\mathrm{CD} 4^{+} \mathrm{CD} 25^{-} \mathrm{T}$ cells and dendritic cells (DC) were purified with purchased reagent kits. The purity of the cells was greater than $98 \%$ as checked by flow cytometry. The T cells were labeled with CFSE (Carboxyfluorescein succinimidyl ester) and cultured with DC ( $\mathrm{T}$ cell: $\mathrm{DC}=10: 1 ; 10^{5} / \mathrm{ml}$ ) in the presence of the specific allergen, OVA, or BSA (control) for 3 days. The CFSE-dilution was assessed by flow cytometry. 

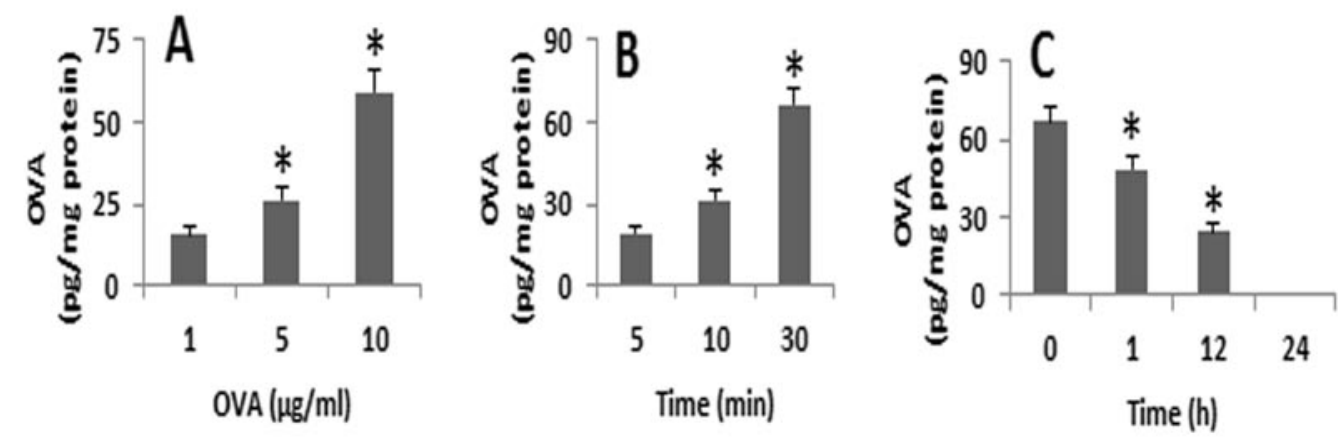

Fig. 1. Gut epithelial cells endocytose and degrade allergens. Caco-2 cells were cultured to monolayers in transwells. The OVA was added to the apical chambers. The monolayers were harvested; the cell extracts were prepared to analyse the contents of the OVA. The bars indicate the levels of OVA in the cell extracts. The data were expressed as mean \pm SD. *, p<0.01, compared with group “ $1 \mu \mathrm{g} /$ ” (A), or group “5min” (B), or group " 0 " (C). The data represent 3 separate experiments.

Statistics

Differences between two groups were analysed using unpaired two-tailed Student's $t$-test, or by oneway analysis of variance (ANOVA) for experimental groups were more than 2 groups. Data were expressed as the mean \pm SD.

\section{Results}

Gut epithelial cells endocytose OVA allergen

Caco- 2 cells were a human colon epithelial cell line; to see if the cells could endocytose exogenous allergens in culture medium, Caco- 2 cells were cultured into a monolayer in the inserts of transwells to confluence. The OVA was added to the apical chambers at graded concentrations. The cells were harvested $30 \mathrm{~min}$ after the addition of the OVA. In separate experiments, Caco- 2 cells were exposed to OVA at $10 \mu \mathrm{g} / \mathrm{ml}$; the cells were harvested at several time points from 1-30 min. The cells were collected; cellular extracts were prepared and analysed by ELISA. The results showed that the OVA was detected in the cell extracts of Caco-2 cells in an OVA dose-(Fig. 1A) and exposure time (Fig. 1B)-dependent manner. The data indicate that the polarized Caco-2 cells can endocytose OVA.

A20 is required in the degradation of endocytic allergens in gut epithelial cells

We next observed the time course that gut epithelial cells degraded the endocytic OVA allergens. We exposed Caco- 2 monolayers to OVA for $30 \mathrm{~min}$; the non-absorbed OVA was washed out with fresh medium. The cells were then cultured with fresh medium for 1-24h. The cells were collected; the cell extracts of Caco-2 cells were analysed by ELISA. The results showed that the contents of OVA in the extracts were gradually reduced; that were below the detectable levels in the samples collected at the $24 \mathrm{~h}$ time point (Fig. 1C). We also did not detect OVA in the basal chambers (data not shown). The results imply that the endocytic OVA is degraded in the polarized Caco- 2 cells within $24 \mathrm{~h}$.

We also assessed the permeability of Caco-2 monolayer to OVA in a $24 \mathrm{~h}$-culture experiment with OVA addition to the apical chamber of transwell; the levels of OVA in the medium taken from the basal chambers of transwell were determined by ELISA. The results showed that low levels of OVA were converted to the basal chambers by medium treated 
Fig. 2. A20 is required in degradation of endocytosed allergen in gut epithelial cells. (A) OVA $(10 \mu \mathrm{g} / \mathrm{ml})$ was added to the apical chambers of transwell (with confluent Caco2 monolayer). Samples were taken from the basal chambers at $1 \mathrm{~h}$, $12 \mathrm{~h}$, and $24 \mathrm{~h}$ respectively. The bars indicate the OVA levels in the culture supernatants in basal chambers. (B) the immune blots show the Caco-2 cell A20 gene knockdown results. Medium: Cells were cultured with medium alone. A20shRNA (cshRNA): Cells were transduced with shRNA of A20 (or control shRNA). (C) the bars indicate the TER of Caco-2 monolayer recorded at the $24 \mathrm{~h}$ time point. The data in bar graphs were presented as mean \pm SD. ${ }^{*}, \mathrm{p}<0.01$, compared with the medium group. The data represent 3 separate experiments.

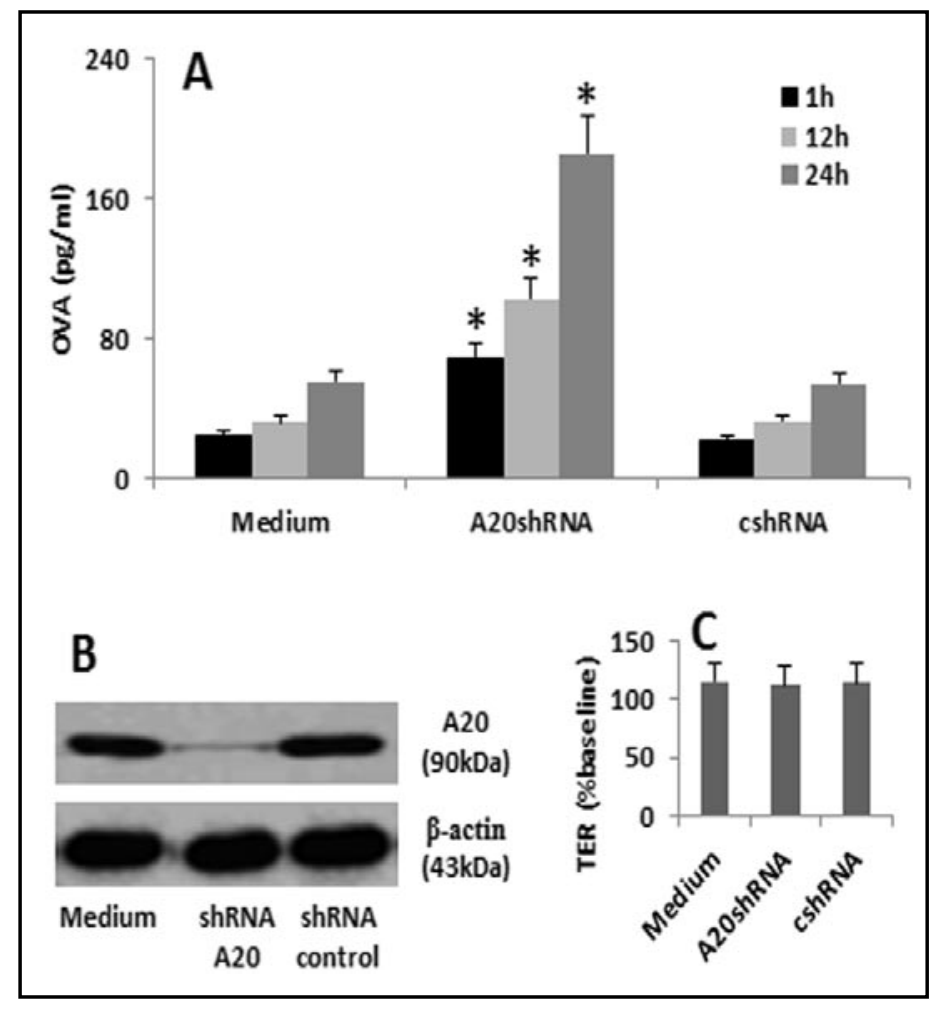

Caco-2 monolayers within 24h. On the other hand, we knocked down the A20 gene from Caco-2 cells; these cells were cultured to monolayers with the TER similar to A20-sufficient monolayers (data not shown). The OVA flux experiments were performed with the A20deficient Caco-2 monolayers. Abundant amounts of OVA were detected in the basal chambers in the course of $24 \mathrm{~h}$ (Fig. 2A-B). To elucidate whether the OVA was transported across the Caco-2 monolayers via the paracellular pathway, we recorded the TER. The results showed that TER at the $24 \mathrm{~h}$ time point was increased about $10 \%$ above the baseline in both A20sufficient and A20-deficient Caco-2 monolayers. There were no significant differences in TER between the A20-sufficient group and A20-deficient group (Fig. 2C). The results indicate that the knockdown of A20 in Caco-2 cells does not affect the paracellular permeability. A20 plays an important role in the degradation of the endocytic OVA in Caco-2 cells.

A20 is required in allergen-carrying endosomes fuse with lysosomes in gut epithelial cells

By immunocytochemistry, we observed OVA-carrying endosomes in Caco-2 cells; some of the OVA-carrying endosomes were fused with lysosomes. In A20-deficient Caco-2 cells, the amounts of OVA-carrying endosome were increased; the positive staining of OVA/ endosome/lysosome was decreased (Fig. 3). The results imply that A20 is required in the endosome/lysosome fusion.

\section{Allergens pass through A20-deficient gut epithelial monolayers conserve antigenicity}

To elucidate if allergens pass through the A20-deficient Caco- 2 monolayer still conserve the antigenicity, we collected peripheral $\mathrm{DC}$ and $\mathrm{CD} 3^{+} \mathrm{CD} 4^{+} \mathrm{CD} 25^{-} \mathrm{T}$ cells from patients with egg allergy history. The T cells were labeled with CFSE and cultured with DC at a ratio of $\mathrm{T}$ cell:DC $=10: 1\left(10^{5} / \mathrm{ml}\right)$ for 3 days. The CFSE-dilution assay was performed with a flow cytometer. The results showed that marked $\mathrm{T}$ cell proliferation was observed in the cells stimulated with the supernatant proteins from A20-deficient group and the commercial OVA; The $\mathrm{T}$ cell proliferation was not different among the cells stimulated with the supernatant proteins from A20-sufficient group, or BSA group, or medium alone. The results indicate that allergens pass through the A20-deficient Caco-2 monolayers still conserve the antigenicity. 


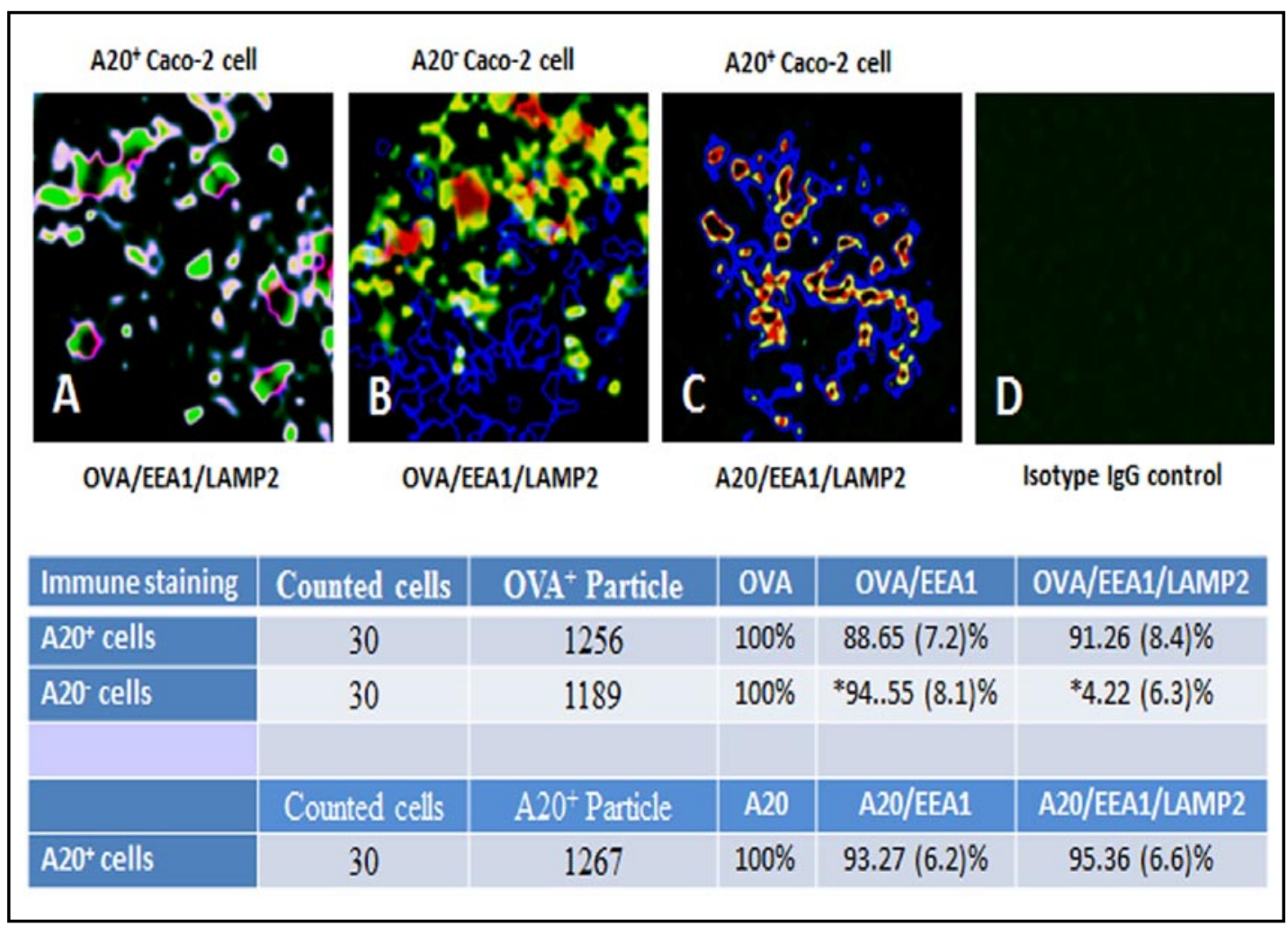

Fig. 3. OVA (or A20)-carrying endosome/lysosome fusion. Caco-2 cells were exposed to OVA for 30min and processed for immune staining. The representative confocal images show the immune positive staining of OVA (or A20; in green), EEA1 (endosome marker; in red) and LAMP2 (lysosome marker; in blue). Each image is one cell. The positive staining particles were counted and presented in the table. The experiment was repeated 3 times.

\section{Discussion}

The present study demonstrates that intestinal epithelial cell line, Caco-2 cells, expresses A20. This is in line with published data that Wang and Vereecke et al reported that A20 was detected in enterocytes in separate studies $[10,15]$. Apart from that those investigators found that A20 played a role in suppression of inflammation in the intestine $[10,15]$, the present data indicate that A20 is a critical molecule in the processing the endocytic allergens in gut epithelial cells. Deficiency of A20 resulted in abundant allergens with strong antigenicity to be transported across the epithelial barrier to reach the basal compartment in transwells via the intracellular pathway. The antigenicity of the transported allergens was reflected by the induction of allergen-specific $\mathrm{T}$ cell proliferation.

Intestinal epithelial barrier dysfunction plays a critical role in a number of diseases; such as food allergy [4] and inflammatory bowel disease [16]. So far the underlying mechanisms are not clear. The present data have provided novel information for this topic. The deficiency of A20 causes the incomplete degradation of the endocytic allergens in intestinal epithelial cells. The un-degraded allergens are transported to the basal compartment of transwells, where is similar to the subepithelial region in the intestine. Our previous work also indicated that under allergic condition, intestinal epithelial barrier dysfunction, a large quantity of allergens were transported to the subepithelial region and the lamina propria $[17,18]$. The present data have further elucidated the pathway by which the allergens with strong antigenicity are transported to the basal compartment via the intracellular route even though the paracellular space is not open which is reflected by the unchanged TER. 


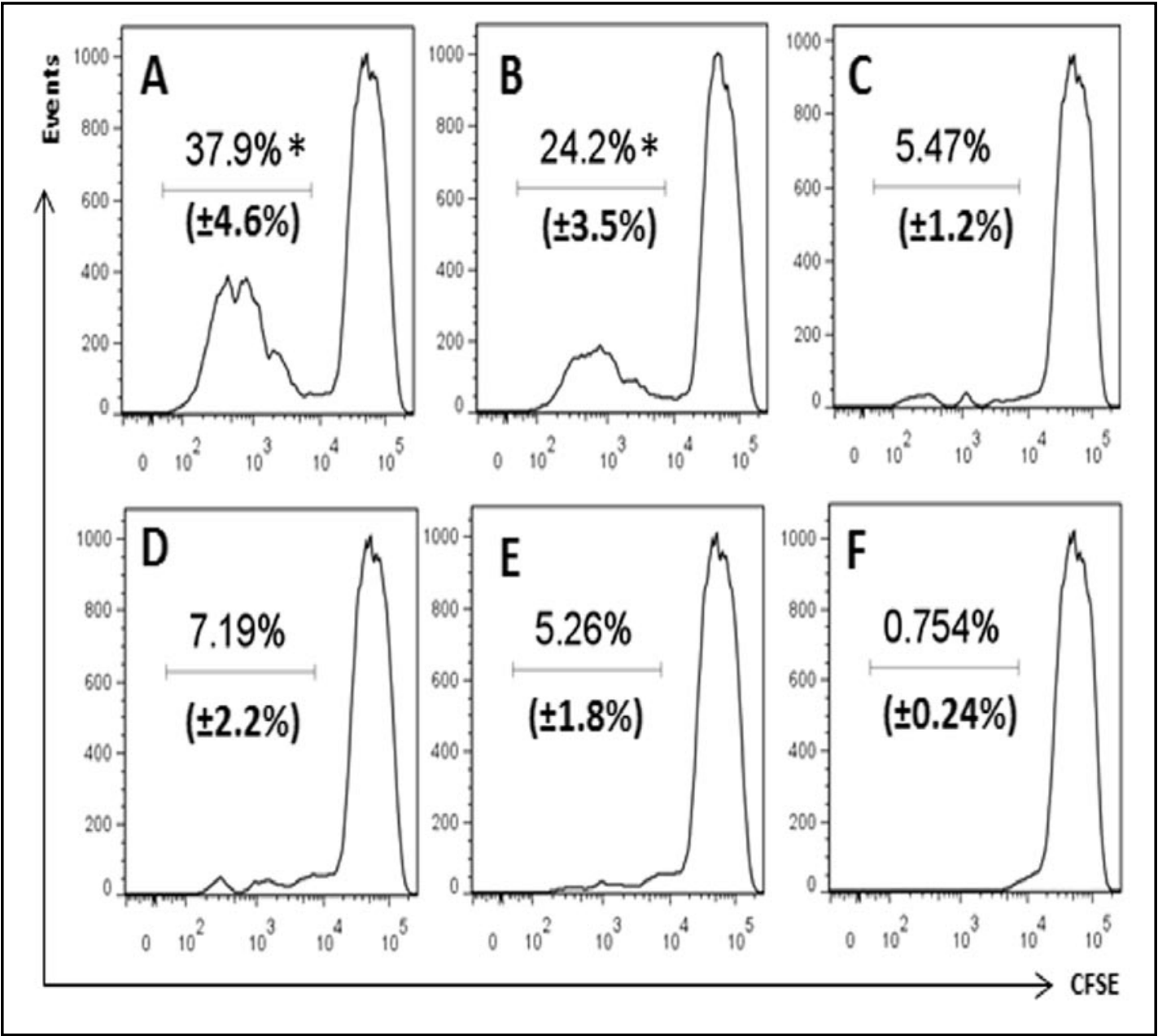

Fig. 4. Antigenicity test. OVA-specific $\mathrm{CD}^{+} \mathrm{CD} 4^{+} \mathrm{CD} 25^{-} \mathrm{T}$ cells and $\mathrm{DC}$ were cultured for 3 days in the presence of commercial OVA (A), or supernatant protein $(10 \mu \mathrm{g} / \mathrm{ml})$ from A20-deficient group (B) or A20sufficient group (C), or medium alone (D), or BSA (E; $10 \mu \mathrm{g} / \mathrm{ml}$ ). Panel F shows the negative staining control. The histograms show the proliferated T cells (the gated cells); the numbers on each panel indicate the mean \pm SD. The data represent 10 separate experiments. ${ }^{*}, \mathrm{p}<0.01$, compared with the medium alone group (D).

The lysosomes contain the acid hydrolase enzymes that can hydrolyze the endocytic allergens, which is dependent on a premise that the allergen-carrying endosomes fuse with lysosomes. Our data are in line with the information that about $90 \%$ OVA-carrying endosomes fused with the lysosomes in Caco-2 cells. Further evidence indicates that in A20-deficient Caco-2 cells, although the OVA-carrying endosomes were abundant in the cytoplasm, less than $5 \%$ of the endosomes had fused with lysosomes. The evidence implies that A20 is required in the endosome/lysosome fusion. The data are in line with published information that A20 contributes to the tethering of endosome and lysosome [12].

Published data indicate that A20 plays a critical role in the prevention of inflammation in the body. One of the features of A20 is that can suppress the activation of NF- $\mathrm{KB}$ and Toll like receptor activities, so as to prevent the gene transcription of a number of proinflammatory cytokines $[19,20]$. Our data have added new information to this point that A20 is also required in the degradation of endocytic allergens in epithelial cells to prevent the allergens to be converted to the basal chambers of transwells, an in vitro experimental model mimicking the subepithelial region in the intestine. 
In summary, the present study reveals that intestinal epithelial cell line, Caco-2 cells, endocytose exogenous allergens, which can be degraded in the epithelial cells. The endosome/lysosome fusion is required in the degradation of endocytic allergens, in which A20 plays a critical role.

\section{Competing financial interests}

The authors declare no competing financial interests.

\section{Acknowledgements}

This study was supported by grants from the Canadian Institutes of Health Research (CIHR \#191063, \#220058, \#177843), Natural Science \& Engineering Research Council of Canada (\#371268), the National Natural Science Foundation of China (\#30971358 and 81061120521) and Shanghai Science and Technology Commission (No. 12XD1404000). P.H., X.R.G., G.Y. and C.C. performed experiments, analyzed data and reviewed manuscript. P.C.Y. and Z.L. designed the project, supervised experiments and wrote the manuscript.

\section{References}

$>1$ Goto Y, Kiyono H: Epithelial barrier: an interface for the cross-communication between gut flora and immune system. Immunol Rev 2012;245:147-163.

-2 Shimizu M: Interaction between Food Substances and the Intestinal Epithelium. Biosci Biotechnol Biochem 2010;74:232-241.

-3 Groschwitz KR, Hogan SP: Intestinal barrier function: Molecular regulation and disease pathogenesis. J Allergy Clin Immunol 2009;124:3-20.

4 Yu LC: Intestinal epithelial barrier dysfunction in food hypersensitivity. J Allergy (Cairo) 2012;2012:596081.

5 Takahashi Y, Nada S, Mori S, Soma-Nagae T, Oneyama C, Okada M: The late endosome/lysosome-anchored p18-mTORC1 pathway controls terminal maturation of lysosomes. Biochem Biophys Res Commun 2012;417:1151-1157.

6 Ganley IG, Wong PM, Jiang X: Thapsigargin distinguishes membrane fusion in the late stages of endocytosis and autophagy. Autophagy 2011;7:1397-1399.

7 Sun T, Wang X, Lu Q Ren H, Zhang H: CUP-5, the C. elegans ortholog of the mammalian lysosomal channel protein MLN1/TRPML1, is required for proteolytic degradation in autolysosomes. Autophagy 2011;7:1308-1315.

-8 Hicke L, Riezman H: Ubiquitination of a Yeast Plasma Membrane Receptor Signals Its Ligand-Stimulated Endocytosis. Cell 1996;84:277-287.

9 Marchese A, Paing MM, Temple BRS, Trejo J: G Protein-Coupled Receptor Sorting to Endosomes and Lysosomes. Annu Rev Pharmacol Toxicol 2008;48:601-629.

-10 Vereecke L, Sze M, Mc Guire C, Rogiers B, Chu Y, Schmidt-Supprian M, Pasparakis M, Beyaert R, van Loo G: Enterocyte-specific A20 deficiency sensitizes to tumor necrosis factor-induced toxicity and experimental colitis. J Exp Med 2010;207:1513-1523.

11 Verstrepen L, Verhelst K, Carpentier I, Beyaert R: TAX1BP1, a ubiquitin-binding adaptor protein in innate immunity and beyond. Trends Biochem Sci 2011;36:347-354.

12 Li L, Soetandyo N, Wang Q Ye Y: The zinc finger protein A20 targets TRAF2 to the lysosomes for degradation. Biochim Biophys Acta 2009;1793:346-353. 
13 Isac SR, Nair GB, Singh DV: Purification and Characterization of Cytotoxin Produced by a Clinical Isolate of Vibrio cholerae 054 TV113. Appl Biochem Biotechnol 2012;167:809-823.

14 Chen X Cho D, Yang PC: Double staining immunohistochemistry. N Am J Med Sci 5 2010;2:241-245.

15 Wang J, Ouyang Y, Guner Y, Ford HR, Grishin AV: Ubiquitin-Editing Enzyme A20 Promotes Tolerance to Lipopolysaccharide in Enterocytes. J Immunol 2009;183:1384-1392.

16 McCole DF: Regulation of epithelial barrier function by the inflammatory bowel disease candidate gene, PTPN2. Ann N Y Acad Sci 2012;1257:108-114.

17 Yang PC, Jury J, Soderholm JD, Sherman PM, McKay DM, Perdue MH: Chronic Psychological Stress in Rats Induces Intestinal Sensitization to Luminal Antigens. Am J Pathol 2006;168:104-114.

-18 Yang PC, Wang CS, An ZY: A murine model of ulcerative colitis: induced with sinusitis-derived superantigen and food allergen. BMC Gastroenterol 2005;5:6.

19 Hitotsumatsu O, Ahmad RC, Tavares R, Wang M, Philpott D, Turer EE, Lee BL, Shiffin N, Advincula R, Malynn BA, Werts C, Ma A: The Ubiquitin-Editing Enzyme A20 Restricts Nucleotide-Binding Oligomerization Domain Containing 2-Triggered Signals. Immunity 2008;28:381-390.

20 Gon Y, Asai Y, Hashimoto S, Mizumura K, Jibiki I, Machino T, Ra C, Horie T: A20 Inhibits Toll-Like Receptor 2and 4-Mediated Interleukin-8 Synthesis in Airway Epithelial Cells. Am J Respir Cell Mol Biol 2004;31:330336. 